\title{
Observations préliminaires sur l'évolution de quelques propriétés d'un sol ferrallitique de Guadeloupe (FWI) après un apport de boues urbaines
}

\author{
M Mench 1, M Clairon 2 \\ avec la collaboration technique de D Nagou 2 \\ 1 INRA, centre de recherches de Bordeaux, station d'agronomie, BP 81, 33883 Villenave d'Ornon cedex; \\ 2 INRA, centre de recherches Antilles-Guyane, station agropédoclimatique des zones caraibes, \\ BP 1232, 97184 Pointe-à-Pitre cedex Guadeloupe, France
}

(Reçu le 9 novembre 1989; accepté le 15 février 1991)

\begin{abstract}
Résumé - Une boue urbaine aérobie a été incorporée aux doses 0,10 et $100 \mathrm{tMS} /$ ha dans l'horizon 0-0,20 $\mathrm{m}$ de parcelles en sol ferrallitique de Guadeloupe. Pour chaque dose, des prélèvements dans l'horizon 0-0,20 m d'une parcelle ont été effectués sur une période de $500 \mathrm{j}$, les mottes de boues de taille supérieure à $5 \mathrm{~mm}$ étant séparées du reste du sol. La caractérisation des échantillons moyens de sol a permis les observations de type indicatif suivantes : - l'amendement $10 \mathrm{t}$ de boues MS/ha se décompose sans qu'on puisse déceler une augmentation du carbone organique du sol, tandis qu'une quantité équivalente à $34 \%$ du carbone apporté par les $100 \mathrm{t}$ de boues MS/ha est encore localisée dans l'horizon $0-0,20 \mathrm{~m}$ après $500 \mathrm{j}$;

- seule la dose $100 \mathrm{t}$ de boues MS/ha entraîne une augmentation de l'azote total du sol, qui représente au terme de l'essai une quantité équivalente à $27 \%$ de l'azote apporté;

- l'acidification du sol est croissante avec les doses de boues : à 100 t MS/ha, le pH diminue de 5,5 à 4,7, ce qui entraîne une augmentation de la teneur en aluminium échangeable;

- l'apport $100 \mathrm{t}$ de boues MS/ha provoque une augmentation de l'humidité à $105^{\circ} \mathrm{C}$ des échantillons à l'état frais ainsi qu'un accroissement de la CEC * du sol;

- à 10 comme $100 \mathrm{t}$ de boues $\mathrm{MS} / \mathrm{ha}$, le bilan des cations échangeables dans l'horizon 0-0,20 m montre des pertes supérieures aux apports.

Les avantages de la boue urbaine utilisée sont sa valeur fertilisante en azote et phosphore, permettant des gains de production végétale, mais ces observations montrent que son application en sol ferrallitique implique aussi, pour certaines propriétés du sol, les mêmes inconvénients qu'une forte fertilisation ammoniacale.
\end{abstract}

sol ferrallitique / boue urbaine / matière organique / pH / cation échangeable / caraïbe

Summary - Preliminary observations on changes in some properties of a ferrallitic soil in Guadeloupe (French West Indies) as a result of sewage sludge application. An aerobic sewage sludge was buried $(0-0.20 \mathrm{~m}$ depth) at 3 levels $(0,10$ and 100 tons DW/ha) in a ferrallitic soil of Guadeloupe (French West Indies) in order to study the biodegradation of the sludge and changes in soil properties. The main characteristics of the soil and the sewage sludge are shown in table l. The study was conducted on plots $\left(20 \mathrm{~m}^{2}\right)$ in field over a period of $500 \mathrm{~d}$. Total rainfall from July 1986 to December 1987 was $4718 \mathrm{~mm}$ and the temperature varied between 22 and $27^{\circ} \mathrm{C}$ (fig 1). The soil was kept vegetation-free and cleared by hand. Samples were taken from the $0-0.20 \mathrm{~m}$ layer and sewage sludge aggregates (diameter $>5 \mathrm{~mm}$ ) were removed by hand. Preliminary observations were reported according to the characterization of the average soil samples.

Changes in the organic carbon and the total nitrogen concentrations in the soil $(0-0.20 \mathrm{~m}$ depth) in relation to the sludge application rate are shown in figure 2. After $500 \mathrm{~d}$, the increase in organic carbon content in the sludged soil $(0-0.20 \mathrm{~m}$ depth) was equivalent to 0 and $34 \%$ of the carbon applied for 10 and 100 tons DW/ha (table 1$)$. In the same manner, for the total nitrogen content the values represented 0 and $27 \%$ of the nitrogen applied (table 1). The cation exchange ability (CEC) (fig 3) and water content (fig 6) of the $100 \mathrm{t} \mathrm{DW/ha} \mathrm{sludged} \mathrm{soil} \mathrm{increased} \mathrm{due} \mathrm{to} \mathrm{the} \mathrm{rise} \mathrm{in} \mathrm{or-}$ ganic carbon content. After 500 d, the difference between the CEC of the control soil and that of the high rate sludged soil was $2 \mathrm{mEq} / 100 \mathrm{~g}$ soil DW. Soil-pH decreased in all plots in relation to sludge application level (fig 4). At $100 \mathrm{t}$ DW/ ha, $p H$ fell from 5.5 to 4.7. Al-exchangeable concentration increased up to $2 \mathrm{mEq} / 100 \mathrm{~g}$ soil $D W$ in the high rate

* CEC : capacité d'échange cationique 
sludged soil due to the extent of $\mathrm{pH}$ decrease (table I). Changes in exchangeable cation $\left(\mathrm{Ca}^{2+}, \mathrm{Mg}^{2+}, \mathrm{K}+, \mathrm{Na}^{+}\right) \mathrm{Con}-$ centrations in the soil (0-0.20 $\mathrm{m}$ depth) are shown in figure 5. The balance-sheet of exchangeable cations generally showed that losses were higher than inputs in the $0-0.20 \mathrm{~m}$ layer, both at the 10 and $100 \mathrm{t}$ DW/ha level (table II). In previous works, the application of this aerobic sewage sludge appeared to be very useful, especially in relation to changes in inorganic nitrogen and phosphorus concentrations in sludged soil, and to its effect on maize crop yield. However, these preliminary observations demonstrated that the sludge application in a ferrallitic soil of Guadeloupe can display the same risks for the soil (ie, soil-pH decrease and rise in Al-exchangeable concentration) as in the case of a high ammoniacal fertilization in which case $\mathrm{pH}$ control (eg liming) would be required.

ferrallitic soil / sewage sludge / organic matter / nitrogen / pH / exchangeable cation / aluminium / Caribbean

\section{INTRODUCTION}

En Guadeloupe, les boues urbaines provenant du traitement des eaux usées constituent une source non négligeable de matière organique, à teneur élevée en azote et en phosphore et encore très peu chargée en micropolluants d'origine industrielle comme les métaux (Giboulot, 1984; Nagou et Clairon, 1985). La principale station (Jarry, Baie Mahault) produit en moyenne 25 $t$ de boues par jour. Ce sont des boues aérobies, pressées au filtre à bandes. Cependant, l'utilisation agricole de ces boues urbaines en zone $\mathrm{Ca}$ raïbe nécessite une étude préalable de leur valeur fertilisante et de leur inocuité pour les sols ou l'environnement.

Les études de la valeur fertilisante ont été entreprises d'abord en sol ferrallitique en raison de la contribution essentielle de la matière organique à sa fertilité (Duchaufour, 1983). Ainsi, Giboulot (1984), Nagou et Clairon (1985) et Clairon et al (1989a) ont démontré que l'application de boues résiduaires urbaines peut remplacer une partie de l'apport d'engrais minéraux nécessaire à une culture de maïs. Dans le sol, l'apport de boues se traduit par une production importante d'azote minéral (Mench et al, 1989) et une augmentation de la teneur de formes organiques et minérales du phosphore (Brossard et al, 1989).

En général, au-delà de la libération d'éléments fertilisants, l'intérêt des amendements réside en leur aptitude à fournir une matière organique stable, susceptible d'améliorer certaines propriétés du sol : capacité de rétention en eau et d'échange de cations, stabilité structurale (Delas, 1971; Linères et al, 1985; Vaughan et Malcolm, 1985; Stevenson, 1986). Pour les boues urbaines, il existe une certaine incertitude car leur composition, à base principalement de protéines et de polysaccharides, diffère de celle des matières organiques issues de résidus végétaux (Juste et Catroux, 1980).

Depuis plus de 20 ans, l'effet des boues urbaines sur la matière organique et les propriétés physico-chimiques des sols est étudié en Europe (Morel et Jacquin, 1980; Guidi, 1981; Catroux et al, 1983; Guckert et Morel, 1983; Linères et al, 1985 , etc). II en ressort par exemple que leur coefficient isohumique est très faible. Cependant, à notre connaissance, très peu d'informations équivalentes ont été recueillies pour les zones tropicales, la Carä̈be en particulier. II nous a donc paru intéressant d'utiliser les échantillons de l'essai Mench et al (1989), pour obtenir des observations sur l'évolution de quelques propriétés d'un sol ferrallitique de zone tropicale humide (Basse-Terre de Guadeloupe, FWI) après un apport de boues urbaines. Nous présentons dans ce travail les variations des teneurs en carbone organique et en azote total, du $\mathrm{pH}$, de la CEC et des teneurs en cations échangeables, sur une période de $500 \mathrm{j}$. Ces résultats sont préliminaires et n'ont pas de valeur statistique éprouvée, car nous ne disposions que des échantillons moyens de sol de 3 parcelles (dose de boues : 0 , 10 et 100 t de matière sèche (MS) / ha).

\section{MATÉRIEL ET MÉTHODES}

\section{Sol}

Les principales caractéristiques du sol sont résumées dans le tableau I. II s'agit d'un sol ferrallitique du Domaine INRA, (Duclos, Guadeloupe, FWI), comportant $52 \%$ d'argiles (avec large dominance de kaolinite et d'halloysite), faiblement désaturé, remanié, hydromorphe sur matériau argileux alluvial. La densité apparente de l'horizon $0-0,2 \mathrm{~m}$ était égale à 0,9 au début et à la fin de l'essai quel que soit le traitement.

\section{Boue urbaine}

Les caractéristiques majeures de la boue utilisée sont mentionnées dans le tableau I. Cette boue est obtenue par un brassage aérobie en bassin et une décantation des eaux usées collectées par la station d'épuration de Jarry (Baie Mahault); après floculation par un 
Tableau I. Caractéristiques de la boue et des sols (horizon 0-0,20 m)

\begin{tabular}{|c|c|c|c|c|c|c|c|}
\hline & \multirow[t]{3}{*}{ Boues } & \multicolumn{6}{|c|}{ Sols } \\
\hline & & \multicolumn{3}{|c|}{$t=0 j$} & \multicolumn{3}{|c|}{$t=512 j$} \\
\hline & & $s_{0}$ & $s_{10}$ & $s_{100}$ & $s_{0}$ & $s_{10}$ & $s_{100}$ \\
\hline C (Anne) (\%) & 38,90 & 1,89 & 1,77 & 1,66 & 1,84 & 1,88 & 2,41 \\
\hline $\mathrm{N}$ total $(\%)$ & 67,80 & 2,04 & 1,93 & 1,74 & 2,00 & 2,08 & 2,79 \\
\hline $\begin{array}{l}\mathrm{C} / \mathrm{N} \\
\mathrm{pH}-\mathrm{eau}\end{array}$ & $\begin{array}{l}5,70 \\
620\end{array}$ & $\begin{array}{l}9,20 \\
5,70\end{array}$ & $\begin{array}{l}9,20 \\
5,60\end{array}$ & $\begin{array}{l}9,50 \\
5,50\end{array}$ & $\begin{array}{l}9,20 \\
5,80\end{array}$ & $\begin{array}{l}9,00 \\
5,30\end{array}$ & $\begin{array}{l}8,60 \\
470\end{array}$ \\
\hline $\mathrm{P}$ Olsen (\%o) & & 0,01 & 0,01 & 0,01 & 0,01 & 0,02 & 0,14 \\
\hline$P$ total $(\% o)$ & 1,60 & & & & & & \\
\hline CEC (mEq/100 g) & & 14,30 & 14,00 & 13,70 & 13,30 & 13,70 & 15,70 \\
\hline Ca éch * $(\mathrm{mEq} / 100 \mathrm{~g})$ & & 7,90 & 7,20 & 6,70 & 7,30 & 5,70 & 4,90 \\
\hline $\mathrm{Mg}$ éch * $(\mathrm{mEq} / 100 \mathrm{~g})$ & & 1,20 & 1,42 & 1,62 & 1,07 & 1,10 & 0,82 \\
\hline $\mathrm{K}$ éch * $(\mathrm{mEq} / 100 \mathrm{~g})$ & & 0,24 & 0,15 & 0,28 & 0,29 & 0,16 & 0,17 \\
\hline $\mathrm{Na}$ éch ${ }^{*}(\mathrm{mEq} / 100 \mathrm{~g})$ & & 0,10 & 0,10 & 0,10 & 0,06 & 0,06 & 0,04 \\
\hline $\mathrm{Al}$ éch * $(\mathrm{mEq} / 100 \mathrm{~g})$ & & 0,20 & 0,30 & 0,40 & 0,40 & 0,60 & 2,00 \\
\hline Ca total ${ }^{* *}(\%)$ & 1,75 & & & & & & \\
\hline $\mathrm{Mg}$ total ${ }^{\star \star}(\%)$ & 0,4 & & & & & & \\
\hline $\mathrm{K}$ total $* *(\%)$ & 0,3 & & & & & & \\
\hline Na total $* \star(\%)$ & 0,21 & & & & & & \\
\hline
\end{tabular}

* extraction $1 \mathrm{~N}$ ammonium acétate $\mathrm{pH} 7$; ** acide fluorhydrique

polymère organique (sans adjonction de calcium ou de fer) la boue est pressée au filtre à bandes.

\section{Dispositif expérimental}

Conçu initialement pour l'étude de l'azote minéral, ce dispositif comprenait 3 parcelles de $20 \mathrm{~m}^{2}$ sans répétition. Elles ont reçu respectivement les doses suivantes : 0,10 et 100 t de boue MS/ha. La boue, à l'état pâteux (17\% MS), a été épandue manuellement sur la surface du sol, en juillet. Après 15 j d'exposition à l'air, elle a été enfouie avec une fraise sur $0,20 \mathrm{~m}$ de profondeur. Le même travail du sol a été effectué dans la parcelle sans apport.

Les précipitations et les températures décadaires pendant la période d'étude sont représentées dans la figure $1 \mathrm{a}$ et $\mathrm{b}$. La pluviométrie cumulée entre l'apport des boues (juillet 1986) et le dernier prélèvement (décembre 1987) a été de $4718 \mathrm{~mm}$. L'étude inclut une période sèche entre le $180^{\mathrm{e}}$ et le $270^{\mathrm{e}} \mathrm{j}$, correspondant à la saison sèche de la zone climatique de janvier à mars. Les températures moyennes décadaires ont varié entre 22 et $27^{\circ} \mathrm{C}$.

\section{Prélèvements et traitement des échantillons de sols}

La date d'épandage des boues a été prise comme temps 0 . Les prélèvements ont été réalisés à la bèche a
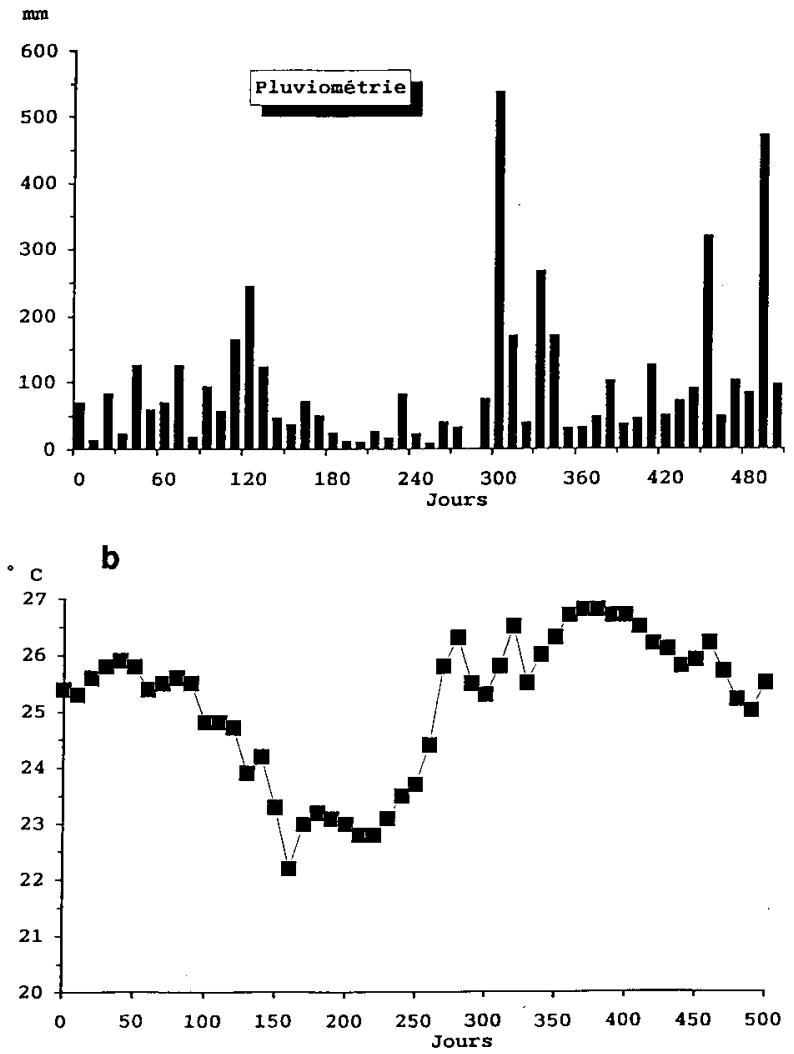

Fig 1. a Pluviométrie = total décadaire du 10 juillet 1986 au 10 décembre 1987 (source : INRA, Domaine expérimental Duclos, Station agropédoclimatique, Guadeloupe, FWI). b Température de l'air : moyenne décadaire du 10 juillet 1986 au 10 décembre 1987 (INRA, station agropédoclimatique). 
dans l'horizon $0-0,20 \mathrm{~m}$, sur une période de $500 \mathrm{j}$. La fréquence des prélèvements a été d'abord hebdomadaire, puis bimensuelle, bimestrielle et enfin trimestrielle. L'échantillon moyen représentatif de chaque traitement a été formé à partir de 4 prélèvements d'environ $1,5 \mathrm{~kg}$ de sol sec, sur lesquels on a effectué au préalable une séparation manuelle des mottes de boues de taille supérieure à $5 \mathrm{~mm}$ de diamètre. On a donc obtenu, à chaque pas de temps, une fraction "boue" (B) et une fraction "sol" (S) qui contient des particules de boue visibles mais non triables car de taille essentiellement inférieure à $2 \mathrm{~mm}$. Ces fractions sont indicées par convention dans le texte en fonction de la dose de boue apportée (exemple, $\mathrm{B}_{10}, \mathrm{~S}_{10}$ ). L'évolution des masses de matière sèche de $B_{10}$ et $B_{100}$ ainsi que leur rapport $C / N$ ont été présentés antérieurement (Mench et al, 1989).

\section{Caractérisation des échantillons moyens de sol}

On a déterminé l'humidité à $105^{\circ} \mathrm{C}$ sur une aliquote, à l'état frais, de chaque échantillon moyen des fractions $S$. Sur les échantillons moyens des fractions $S_{0}, S_{10}$ et $S_{100}$, séchés à l'air, on a procédé aux déterminations suivantes : $\mathrm{pH}$ dans l'eau distillée (rapport 1/2,5), CEC par extraction à l'acétate d'ammonium $1 \mathrm{~N} \mathrm{pH} 7$ (Bonneau et Souchier, 1979) et teneurs en cations échangeables $\left(\mathrm{Ca}^{2+}, \mathrm{Mg}^{2+}, \mathrm{Na}^{+}, \mathrm{K}^{+}\right)$dans les extraits acétate d'ammonium par spectrophotométrie en flamme, carbone organique (Anne, 1945), azote total (minéralisation Kjeldahl, catalyseur acide salicylique, Bradstreet, 1965). Le mode d'échantillonnage n'a pas permis une étude de la variabilité de ces paramètres.

\section{RÉSULTATS}

\section{Carbone organique et azote total dans les fractions $S$ (sol)}

Sur la période d'étude, les teneurs moyennes en carbone organique et en azote total de $S_{0}$ sont égales à $1,83 \%$ et $0,20 \%$. Pour ces 2 paramètres, le coefficient de variation est de $3 \%$. La figure 2 montre l'évolution du carbone organique et de l'azote total dans les fractions $S_{0}, S_{10}$ et $S_{100}$ sur $500 \mathrm{j}$. Seule la dose $100 \mathrm{t}$ MS de boues/ ha se traduit par une augmentation des teneurs en carbone organique et en azote total dans la fraction S. Au-delà de $40 \mathrm{j}$, l'allure globale des courbes, relatives aux teneurs en carbone organique et en azote total de $S_{100}$, est décroissante, avec des dents de scie dont la signification ne peut être définie sur les seuls échantillons moyens en notre possession. Au terme de $500 \mathrm{j}$, la différence entre les teneurs finale et initiale (tableau I) permet d'établir qu'une quantité de a

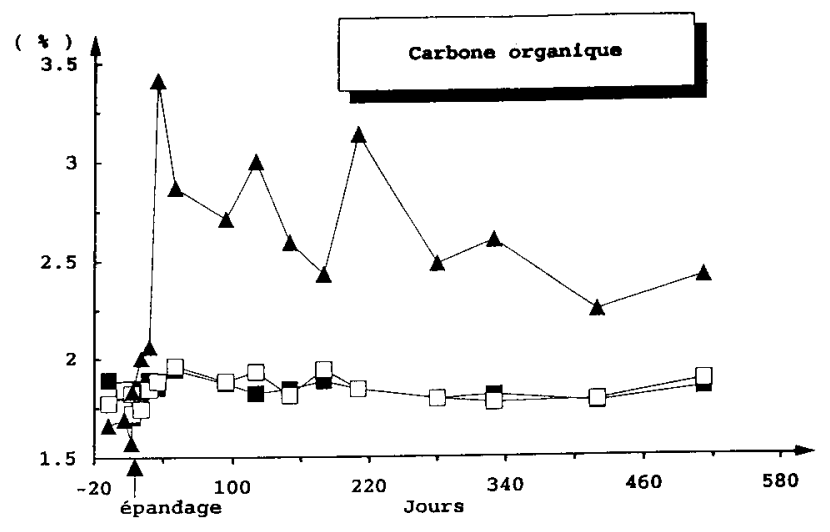

b

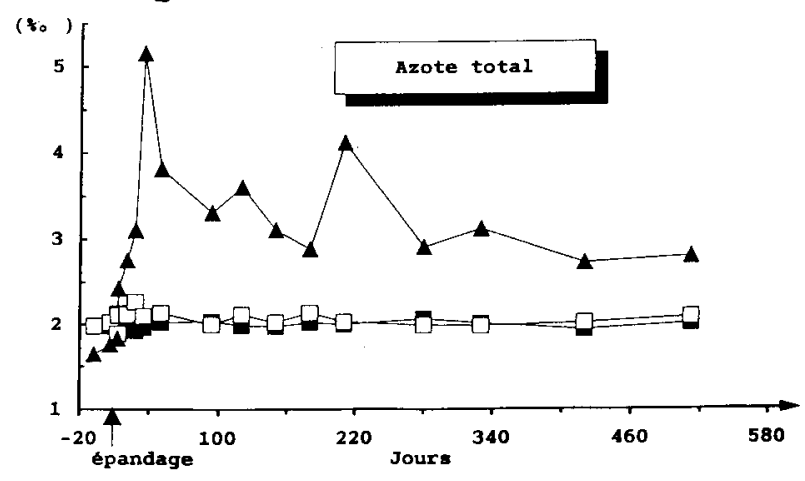

Fig 2. Évolution a de la teneur en carbone organique (\%) et b en azote total (\%) dans les fractions $S: S_{0}=; S_{10} \square$; $\mathrm{S}_{100} \Delta$.

carbone équivalente à $34 \%$ du carbone organique apporté par la dose 100 t MS/ha est localisée dans la fraction $S_{100}$ de l'horizon 0-0,20 m. Le calcul réalisé pour l'azote total fournit une valeur de $27 \%$.

\section{Capacité d'échange de cations, CEC (fig 3)}

Pour $S_{0}$ et $S_{10}$, la CEC est pratiquement constante tout au long de l'essai (moyennes respec-

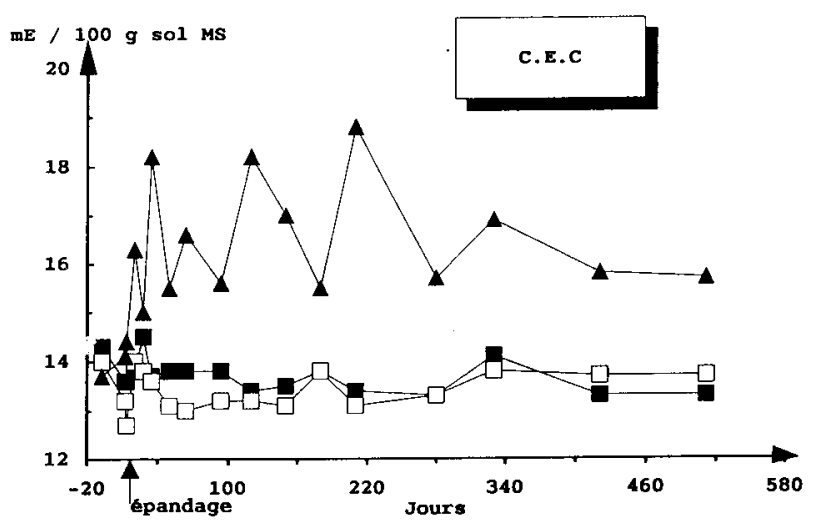

Fig 3. Évolution de la capacité d'échange des cations (CEC) des fractions $\mathrm{S}: \mathrm{S}_{0} \mathbf{m} ; \mathrm{S}_{10} \square ; \mathrm{S}_{100} \Delta$. 
tives : 13,7 et $13,4 \mathrm{mEq} / 100 \mathrm{~g}$, coefficient de variation de 2,3 et $2,9 \%$ ). Par contre, l'évolution de la CEC pour $S_{100}$ montre globalement une augmentation sur les 40 premiers jours, puis des variations en dents de scie autour d'une valeur de $17 \mathrm{mEq} / 100 \mathrm{~g} \mathrm{sol} \mathrm{sec}$. Au terme de l'essai, la différence entre les CEC de $S_{0}$ et $S_{100}$ est de 2 $\mathrm{mEq} / 100 \mathrm{~g} \mathrm{sol} \mathrm{sec}$.

En ce qui concerne la saturation du complexe d'échange, le tableau I montre qu'elle était initialement de l'ordre de $63 \%\left(S_{0}\right.$ et $\left.S_{10}\right)$ et $59 \%$ $\left(S_{100}\right)$. Après $500 \mathrm{j}$, sa valeur est égale à $65 \%$ $\left(S_{0}\right), 51 \%\left(S_{10}\right)$ et $38 \%\left(S_{100}\right)$. Les apports de boues entraînent donc une diminution du taux de saturation.

\section{pH (fig 4)}

L'acidification des fractions $S$ croît avec les doses d'apport de boues. Le $\mathrm{pH}$ de $S_{10}$ diminue pendant $220 \mathrm{j}$ puis évolue parallèlement à celui de $S_{0}$ avec une différence de 0,5 unité $\mathrm{pH}$. On constate 2 phases d'évolution pour le $\mathrm{pH}$ de $S_{100}$ : tout d'abord une augmentation aussitôt après l'enfouissement des boues (15e j), puis une diminution rapide de 1 unité $\mathrm{pH}$ en $45 \mathrm{j}$. Après $500 \mathrm{j}$, le pH de $S_{100}$ égale 4,7 soit une différence de 0,8 unité avec la valeur initiale.

\section{Teneurs en cations échangeables (fig 5)}

Les modifications sont bien sûr plus marquées pour $S_{100}$ que pour $S_{10}$. L'enfouissement de la dose $100 \mathrm{t} \mathrm{MS/ha}$ entraine une augmentation rapide des teneurs en cations échangeables : les maximums observés à $40 \mathrm{j}$ sont équivalents à $1620 \mathrm{~kg} \mathrm{Ca}, 432 \mathrm{~kg} \mathrm{Mg}, 491 \mathrm{~kg} \mathrm{~K}$ et $207 \mathrm{~kg} \mathrm{Na}$ à

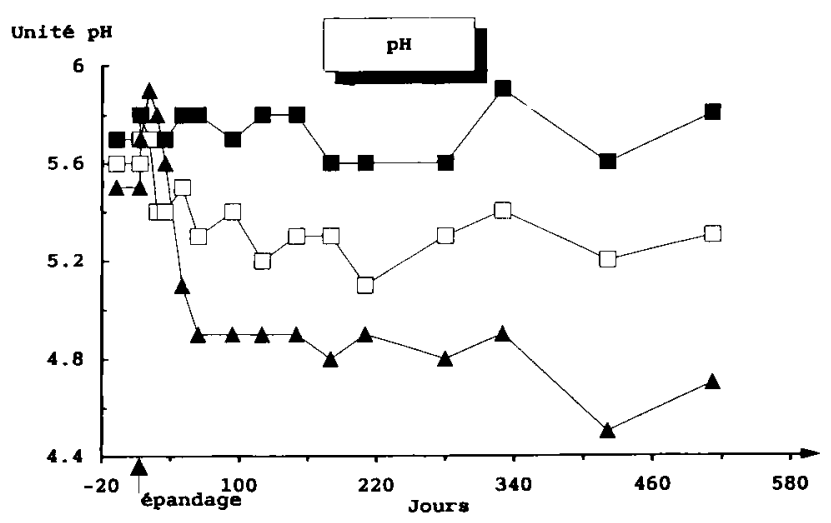

Fig 4. Évolution du $\mathrm{pH}$ des fractions $\mathrm{S}: \mathrm{S}_{0} \square ; \mathrm{S}_{10} \square ; \mathrm{S}_{100} \boldsymbol{A}$. l'ha. Ces valeurs sont sensiblement égales aux apports, sauf pour $\mathrm{K}$ où la teneur est supérieure. Au-delà de 40 j, l'allure globale des courbes relatives aux teneurs en cations échangeables dans $\mathrm{S}_{100}$ est décroissante. Le sodium est le plus vite lessivé hors de l'horizon 0-0,20 m. Après $500 \mathrm{j}$, les teneurs en cations échangeables de $S_{10}$ et $S_{100}$ sont inférieures ou égales à celles de $S_{0}$.

Le bilan en cations échangeables établi pour les fractions $S$ de l'horizon 0-0,20 m montre que les pertes sont en général supérieures aux apports et augmentent avec les doses croissantes de boues (tableau II).

\section{Aluminium échangeable}

L'aluminium échangeable a été déterminé au début et à la fin de l'essai. On constate une augmentation de la teneur en aluminium échangeable dans les fractions $S_{10}$ et $S_{100}$ (tableau I).

\section{Humidité à $105^{\circ} \mathrm{C}(\theta)$}

L'humidité des fractions $S$ dépend bien entendu des conditions climatiques dans la période qui précède l'échantillonnage. Cependant, alors que $\theta_{0}$ et $\theta_{10}$ sont identiques tout au long de l'essai (20-30\% par rapport au poids sec), $\theta_{100}$ est supérieur à $\theta_{0}$ (écart de 3 à $10 \%$ du poids sec pendant 240 j). Nous avons remarqué qu'il existe

Tableau II. Bilan des cations échangeables des fractions $S$ de l'horizon $0-0,20 \mathrm{~m}$ ( $T$ : gain ou pertes après $500 \mathrm{j} ; \mathrm{A}$ : apport)

\begin{tabular}{lrrrr}
\hline & & \multicolumn{3}{c}{ en kg.ha-1 } \\
\cline { 2 - 5 } & & $S_{0}$ & $S_{10}$ & \multicolumn{1}{c}{$s_{100}$} \\
\hline \multirow{2}{*}{$\mathrm{Ca}^{2+}$} & $\mathrm{A}$ & 0 & 175 & 1750 \\
& $\mathrm{~T}$ & -216 & -715 & -2398 \\
$\mathrm{Mg}{ }^{2+}$ & $\mathrm{A}$ & 0 & 40 & 400 \\
& $\mathrm{~T}$ & -28 & -108 & -573 \\
$\mathrm{~K}+$ & $\mathrm{A}$ & 0 & 30 & 300 \\
& $\mathrm{~T}$ & +35 & +7 & -377 \\
$\mathrm{Na}^{+}$ & $\mathrm{A}$ & 0 & 21 & 210 \\
& $\mathrm{~T}$ & -16 & -37 & -235 \\
\hline
\end{tabular}



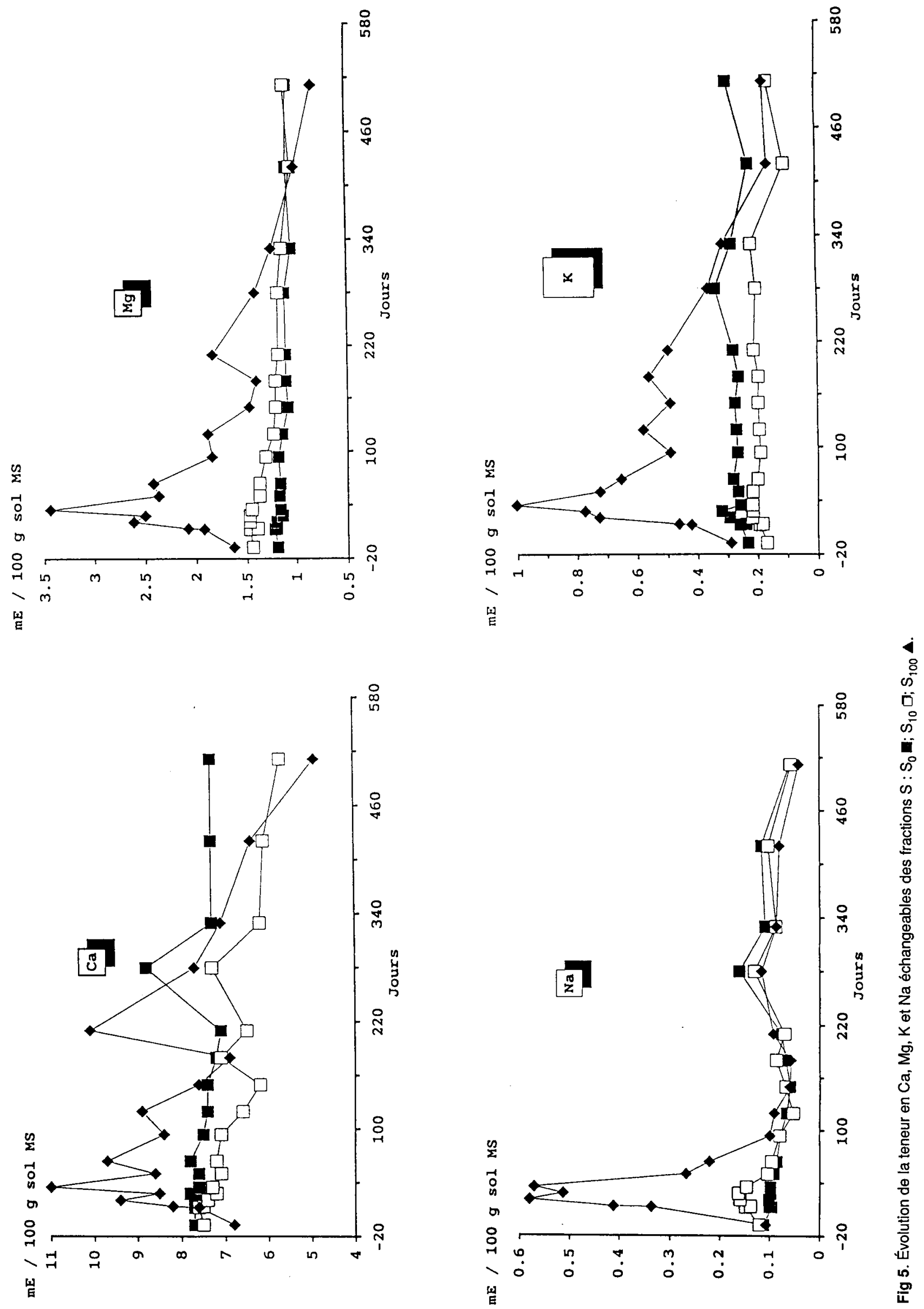
une relation significative à $1 \%$ entre la teneur en carbone de la fraction $S_{100}$ et son humidité $\left(\theta_{100}\right.$ $=4,2^{*} \mathrm{C}_{100}+22,7 ; r=0,66$ ) (fig 6 ). Cette relation est légèrement améliorée si l'on considère la variation de $\left(\theta_{100}-\theta_{0}\right)$, pour, en première approximation, limiter l'effet ponctuel des conditions climatiques, en fonction de $\left(\mathrm{C}_{100}-\mathrm{C}_{0}\right)\left(\left[\theta_{100}-\theta_{0}\right]=\right.$ $\left.3,9^{*}\left[C_{100}-C_{0}\right]+2,78 ; r=0,72\right)$.

\section{DISCUSSION}

Cette étude présente des observations complémentaires aux travaux antérieurs sur la valorisation de boues urbaines dans les sols ferrallitiques de la Guadeloupe (Clairon et al, 1989a; Mench et al, 1989; Brossard et al, 1989). Cependant, celles-ci ont un caractère préliminaire car, ne disposant que des échantillons moyens des fractions $\mathrm{S}$ (sol après tri des mottes de boues) de 3 parcelles, nous n'avons pu estimer la variabilité des paramètres.

La comparaison des sols ayant reçu les doses 0,10 et 100 t de boues MS/ha a permis d'observer des variations pour les caractéristiques suivantes des fractions $S$ : teneurs en carbone organique et azote total, CEC et teneurs en cations échangeables, $\mathrm{pH}$, aluminium échangeable, humidité à $105^{\circ} \mathrm{C}$.

L'évolution des masses (MS) des mottes de boues avait montré que $B_{10}$ (fraction «boues» après tri du sol ayant reçu $10 \mathrm{t}$ de boues $\mathrm{MS} / \mathrm{ha}$ ) disparaît en $110 \mathrm{j}$ et que $\mathrm{B}_{100}$ (fraction «boues» après tri du sol ayant reçu 100 t de boues MS/ha) diminue rapidement $(-80 \%)$ en 200 j, puis décroît lentement sur les 300 autres (Mench et al, 1989). La mesure du carbone organique des fractions $S_{10}$ et $S_{100}$ montre que :

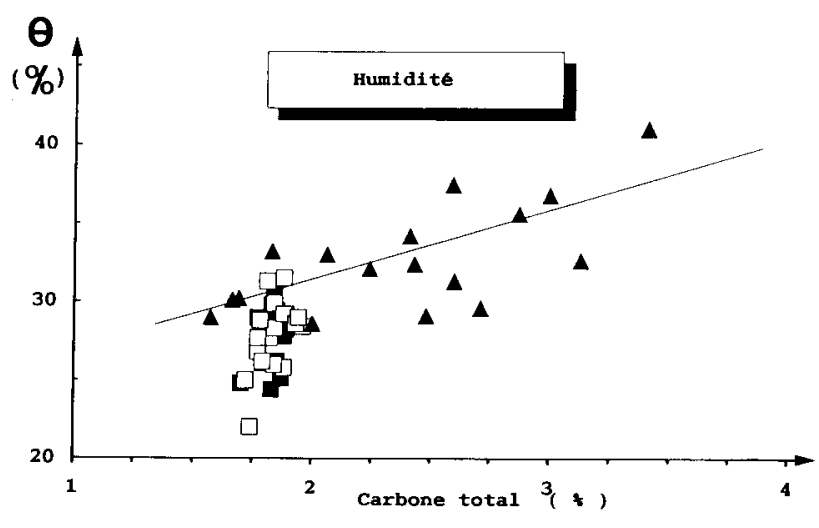

Fig 6. Distribution de l'humidité à $105^{\circ} \mathrm{C}$ (\% du poids sec de sol) dans les échantillons moyens des fractions $S$ à l'état frais, en fonction de leur teneur en carbone organique (Anne) : $\mathrm{S}_{0} \mathbf{\square} ; \mathrm{S}_{10} \square ; \mathrm{S}_{100} \boldsymbol{A}$.
- $B_{10}$ s'est décomposé, sans que nous ayons pu déceler une augmentation du stock organique de $\mathrm{S}_{10}$;

- la diminution de la masse (MS) de $B_{100}$ s'est accompagnée d'une augmentation de la teneur en matière organique de $S_{100}$, le maximum étant atteint $40 \mathrm{j}$ après l'épandage; au-delà, la teneur en matière organique de $S_{100}$ a diminué, les transferts ne compensant sans doute plus sa décomposition. Les mécanismes de ce transfert (fragmentation des mottes de boues, remaniement de la matière organique exogène par les microorganismes, etc) restent à définir.

De toute évidence, la production d'azote minéral et les transferts de matière organique (particulaire ou non) après l'apport de boues sont 2 éléments majeurs à l'origine de la différenciation des fractions $S$.

Les 2 phases de l'évolution du $\mathrm{pH}$ de $S_{100}$ coïncident dans le temps avec les séquences successives de production de $\mathrm{NH}_{4}+(1000 \mathrm{~kg}$ $\mathrm{N} / \mathrm{ha}$ ) et de nitrification décrites par Mench et al (1989). L'ammonification d'azote organique, puis sa nitrification apparaissent comme le mécanisme le plus probable de l'acidification de $S_{10}$ et surtout de $S_{100}$. À cela s'ajouterait la production de $\mathrm{CO}_{2}$ (microorganismes, oxydations, etc) et la synthèse de composés organiques acides. Ces 2 derniers processus jouent vraisemblablement un rôle secondaire car, dans des situations analogues d'apports, massifs (composts urbains 120 tha) ou répétés (fumier, bagasse), de matériaux ayant un $\mathrm{C} / \mathrm{N}$ plus élevé que les boues, le $\mathrm{pH}$ en sol ferrallitique n'est pas modifié (Clairon et Nagou, 1978; Clairon et al, 1980, 1989b).

L'acidification a pour conséquence une augmentation de l'aluminium échangeable (tableau I). II en résulte un risque de phytotoxicité, possible par exemple chez le maïs cultivé en sol acide dès que l'aluminium échangeable excède $0,55 \mathrm{mEq} / 100 \mathrm{~g}$ de sol (Juste, 1978). Cette situation, souvent conjuguée avec une atrophie des racines, peut également être à l'origine d'une moindre disponibilité d'éléments nutritifs pour les végétaux (exemple: $\mathrm{Mg}, \mathrm{P}$ ) (Juste, 1978).

La CEC et l'humidité à $105^{\circ} \mathrm{C}$ ont été modifiées par l'apport $100 \mathrm{t}$ MS/ha. Les variations en dents de scie de la CEC de $S_{100}$ peuvent être dues à une variabilité d'échantillonnage ou à une augmentation, soit des fines particules de boues, soit de la biomasse microbienne. La relation entre CEC et teneur en carbone organique ou azote total, établie de longue date (Delas, 1971), apparaît lorsque l'on compare les figures 2 et 3 . L'origine et la disponibilité de l'eau sup- 
plémentaire dans $S_{100}$, par rapport à $S_{0}$, ou $S_{10}$, doivent être précisées.

Dès l'enfouissement des boues, une grande partie des cations échangeables migre des fractions $B$ vers les fractions $S$, soient pour 10 et $100 \mathrm{t} \mathrm{MS} / \mathrm{ha}: 61$ et $30 \%$ du $\mathrm{Ca}, 38$ et $25 \%$ du $\mathrm{Mg}, 53$ et $42 \%$ du K, 71 et $61 \%$ du Na. Après 60 j, la presque totalité des cations apportés par $100 \mathrm{t} \mathrm{MS/ha} \mathrm{est} \mathrm{contenue} \mathrm{dans} \mathrm{la} \mathrm{fraction} S_{100}$. Le transfert des cations en solution serait plus important par rapport au transfert avec les particules de boues, sauf dans le cas du calcium. En effet, la comparaison à $210 \mathrm{j}$ de l'évolution du stock de matière organique et des teneurs en cations échangeables montre que $75 \%$ du carbone apporté par $100 \mathrm{t} \mathrm{MS/ha}$ est encore présent dans I'horizon $0-0,20 \mathrm{~cm} \quad\left(B_{100}=2 \%, S_{100}=\right.$ $73 \%)$, tandis que les teneurs en cations représentent respectivement $\mathrm{Ca}=70 \%, \mathrm{~K}=50 \%, \mathrm{Mg}$ $=11 \%$ et $\mathrm{Na}=0 \%$ des apports. L'entraînement des cations en profondeur est difficile à apprécier en parcelles mais justifie une étude en lysimètre. Par ailleurs, la teneur maximale en $\mathrm{K}$ échangeable observée dans $S_{100}$ est supérieure à la quantité de K apportée par les boues (fig 5). Ce potassium pourrait être libéré par les minéraux argileux accessoires de type 2:1, présents dans les sols ferrallitiques d'origine volcanique en Guadeloupe (Van Oort, 1988), à la suite de l'acidification de $S_{100}$. II est probable que ces minéraux, qui dans des situations analogues montrent une affinité potassique (Delvaux et al, 1988), interviennent sur la rémanence de $K$ dans les fractions S jusqu'au $220 \mathrm{e}$ j.

\section{CONCLUSION}

L'attrait d'un apport de boues urbaines en sol ferrallitique de zone tropicale humide réside dans la production d'azote minéral (Mench et al, 1989), l'augmentation des teneurs du sol en phosphore organique et minéral (Brossard et al, 1989) et les gains de production végétale : en expérimentation de longue durée, les meilleurs rendements de maïs ont été obtenus avec $100 \mathrm{t} \mathrm{MS} / \mathrm{ha}$ pour 2 ans, associés toutefois à des chaulages de correction (Nagou et Clairon, 1985; Clairon et al, 1989a). Cependant, nos observations préliminaires sur l'évolution de quelques autres caractéristiques du sol soulignent la nature complexe de l'incidence d'un apport de boues. L'acidification du sol, l'augmentation de l'aluminium échangeable, le lessivage des cations sont des éléments porteurs de risques, qui s'ajoutent à l'incertitude sur le devenir de l'azote minéral. En lysimètres, on a constaté un lessivage important des nitrates (Mench et al, 1989). Une réserve s'impose donc dès l'application de 10 t.ha-1 $^{-1}$.an-1 (MS). En général, les apports de boues sont raisonnés sur l'azote disponible et cette dose apporte déjà 2 fois la fertilisation courante en azote du maïs. D'autre part, le stock organique, la teneur en eau et la CEC du sol ne pourraient être améliorés que par des apports massifs, dont les avantages par exemple pour 100 t.ha-1. $^{-1}$ an $^{-1}$ (MS) se sont estompés après $500 \mathrm{j}$ et qui ont accentué les inconvénients précités. Pour mieux raisonner les doses de boues à appliquer et la fréquence de leur épandage en sol ferrallitique de zone tropicale humide, il faut maintenant rechercher les seuils d'action, sur le comportement du sol et le fonctionnement des végétaux, et établir les pratiques culturales à associer (exemple : chaulage d'entretien, complément minéral, etc). II serait souhaitable, par ailleurs, de modifier la filière de traitement des boues (procédé de digestion ou chaulage avant pressage), ce qui pourrait amener à revoir ces conclusions.

\section{RÉFÉRENCES}

Anne $P$ (1945) Sur le dosage rapide du carbone organique des sols. Ann Agron Paris 15, 161-172

Bonneau M, Souchier B (1979) Pédologie, tome 2 : Constituants et propriétés du sol. Masson, Paris

Bradstreet RB (1965) The Kjeldahl method for organic nitrogen. Acad Press, New-York, Londres

Brossard M, Mench M, Clairon M (1989) Effet d'un apport de boue urbaine sur l'évolution à court terme des formes du phosphore d'un sol ferrallitique. $C R$ CFCS Guadeloupe (sous presse)

Catroux G, L'hermite P, Suess E (1983) The influence of sewage sludge application on physical and biological properties of soil. Commission of the European Communities, Munich, Germany, 1981/06/2324. D Reidel Publ Dordrecht (NL)

Clairon M, Nagou D (1978) Emploi de certains résidus urbains en culture intensive sous climat tropical. Nouv Agron Antilles-Guyane 4, 237-246

Clairon M, Nagou D, Sobesky O (1980) Amendements organiques et cultures intensives sur sol ferrallitique acide en zone tropicale humide. Doc. ronéo, Station d'Agronomie, INRA Antilles-Guyane

Clairon M, Giboulot MC, Sobesky O (1989a) Effets d'apports de boues activées sur maïs et sur sol ferrallitique. CR CFCS Guadeloupe (sous presse)

Clairon M, Picard D, Nagou D, Sobesky O (1989b) Effet fertilisant du compost d'ordures ménagères en milieu tropical sur 3 terres (plante-test : aubergine, laitue). CR CFCS Guadeloupe (sous presse)

Delas J (1971) Évolution des propriétés d'un sol sableux sous l'influence d'apports massifs et répétés 
de matières organiques de différentes origines. Ann Agron Paris 22, 585-610

Delvaux B, Herbillon A, Dufey J, Burtin G, Vielvoye L (1988) Adsorption sélective du potassium par certaines halloysites (10 $\AA$ ) de sols tropicaux développés sur roches volcaniques. Signification minéralogique. CR Séances Acad Sci, série // 307, 311-316

Duchaufour $\mathrm{P}$ (1983) Pédologie, tome 1 : Pédogenèse et classification. 2e édition, Masson, Paris

Giboulot MC (1984) Effets d'apports de boues résiduaires de stations d'épuration en sol ferrallitique tropical : modifications révélées par le comportement d'un peuplement de maïs. Mémoire DAA, INA $P G, 53 p$

Guckert A, Morel JL (1983) Influence des apports de déchets sur les sols et les végétaux. Rech Environ 26, 378-395

Guidi G (1981) Relationship between organic matter of sewage sludge and physico-chemical properties of soil. In : Characterization, treatment and use of sewage sludge. Comm Eur Communities, Vienna, 1980/10/21-23. D Reidel Publ Co, Dordrecht (NL) $530-544$

Juste C (1978) La culture du maïs en terres acides. Perspect Agric 17, 29-33

Juste C, Catroux G (1980) Intérêt agronomique des boues résiduaires et conditions préalables à leur utilisation. In : Séminaire EAS Utilisation agricole des boues provenant des stations d'épuration, Bâle, 1980/09/24-26, 1-24
Linères $M$, Juste $C$, Tauzin J, Gomez A (1985) Effect of a long term sludge disposal on the soil organic matter characteristics. In : Proc 4 th Int Symp Processing and use of organic sludge and liquid agricultural wastes (P L'hermite ed) Rome, 1985/10/ 08-11, Reidel Publ Co, Dordrecht (NL), 290-303

Mench M, Clairon M, Sobesky O, Nagou D (1989) Dynamique en temps court de l'azote minéral en sol ferrallitique nu après apport d'une boue urbaine. Agronomie 9, 785-793

Morel JL, Jacquin F (1980) Influence des boues des stations d'épuration sur les propriétés physiques du sol. In : Séminaire EAS Utilisation agricole des boues provenant des stations d'épuration, Bâle, 1980/09/24-26, 1-11

Nagou D, Clairon M (1985) Et si ... l'agriculture guadeloupéenne recyclait les déchets ? Exemple des boues urbaines. Bull Agron Antilles-Guyane 3, 1-3

Stevenson FJ (1986) Cycles of soil : carbon, nitrogen, phosphorus, sulfur, micronutrients. John Wiley \& Sons, New-York

Van Oort F (1988) Présence et évolution des minéraux argileux accessoires de type 2:1 dans les sols ferrallitiques d'origine volcanique de la Guadeloupe. Conséquence physico-chimiques. $C R$ Séances Acad Sci, sér I/ 307, 1297-1302

Vaughan D, Malcolm RE (1985) Soil organic matter and biological activity. Developments in plant and soil sciences, 16. Martinus Nijhoff/Dr W Junk Publ, Dordrecht (NL) 